\title{
Dynamic recrystallization during high-strain-rate tension of copper
}

N. Mortazavi ${ }^{a^{*}}$, N. Bonora $^{b}$, A. Ruggiero ${ }^{b}$ and M. Hörnqvist Colliander ${ }^{a}$

${ }^{\mathrm{a}}$ Department of Physics, Chalmers University of Technology, Gothenburg, Sweden

${ }^{\mathrm{b}}$ Civil and Mechanical Engineering, University of Cassino and Southern Lazio, Cassino, Italy

*Corresponding author: magnus.colliander@chalmers.se

\begin{abstract}
Discontinuous dynamic recrystallization can occur during dynamic tensile extrusion of copper, which is subjected to uniaxial tensile strains of $\sim 5$ and strain rates up to $10^{6} \mathrm{~s}^{-1}$ in the extruded section. Through high-resolution transmission Kikuchi diffraction we show that nucleation occurs through subgrain rotation and grain boundary bulging at boundaries between $\langle 001\rangle$ and $\langle 111\rangle$ oriented grains. The observed nuclei consist of subgrains with a size of approximately $200-400 \mathrm{~nm}$.
\end{abstract}

\section{Keywords:}

The term recrystallization refers to a process where a deformed microstructure is replaced by new defect-free grains in order to decrease the stored energy from plastic deformation. When recrystallization occurs during ongoing deformation it is referred to as dynamic recrystallization (DRX), which is an important phenomenon in many metal working processes, such as hot rolling, extrusion and forging, as well as during creep deformation [1]. DRX can occur in a continuous or discontinuous fashion, depending on the material and deformation mode. The continuous process involves rotation of subgrains, which occurs simultaneously in the entire microstructure, whereas discontinuous DRX involves an inhomogeneous nucleation step followed by growth of the recrystallized grains by high angle grain boundary (HAGB) migration [1]. Moreover, continuous DRX is favored by a low stacking fault energy (SFE) due to the easier cross-slip and recovery, whereas a high SFE typically results in a discontinuous process.

DRX can also occur under high-strain-rate conditions, typically for combinations of shear strains 
exceeding around 3 , shear strain rates above $10^{4} \mathrm{~s}^{-1}$ and homologous temperatures above approximately $0.4-0.5 T_{m}$ [2]. For practical reasons, dynamic testing is usually performed in shear [3-6] or shear-compression [7]. Under such loading conditions DRX occurs locally through a mechanism similar to the continuous process described above, involving the formation of elongated subgrains or bands, which break up into an equiaxed structure by local rotation of subgrain walls [3]. This takes place also in high SFE materials such as copper, due to the localization of the plastic deformation into shear bands resulting from the adiabatic heating and/or geometrical constraints [3]. Through the introduction of a new test method, called dynamic tensile extrusion (DTE), very high strains and strain rates can be now achieved in uniaxial tension [8]. Recently, it has been shown that DRX in oxygen-free high conductivity (OFHC) copper could be obtained in the uniaxially extruded section of a DTE tested specimen [9], where tensile strains in the order of 5 were reached in combination with strain rates of around $10^{6} \mathrm{~s}^{-1}$ and quasi-adiabatic heating to temperatures exceeding $0.75 T_{m}$. Although grain growth primarily occurred during post-test cooling of the specimen in the die, the nucleation was suggested to occur during deformation since the kinetics of static recrystallization is too slow. The occurrence of DRX could potentially suppress necking and thus be expected to increase the achievable jet elongation [10]. On the other hand, Rittel et al. [7, 11, 12] have suggested that DRX can lead to strain localization into shear bands, which in turn could initiate premature failure. As localized shear bands has previously been observed in fragments of DTE tested copper [8], although the relation to ductility was not further investigated, the occurrence of shear localization under dynamic (nominally) tensile deformation cannot be neglected. Consequently, there is great interest in understanding DRX under dynamic tensile loading in order to develop accurate predictive models for its onset, and subsequent effect on the deformation.

In the present paper we investigate the possible DRX mechanism during DTE of OFHC copper with random starting texture through electron backscatter diffraction (EBSD) and high-resolution orientation mapping by means of transmission Kikuchi diffraction (TKD). TKD, implemented in a field emission gun scanning electron microscope (FEG-SEM) is capable of improving the spatial resolution of the EBSD technique to around 2-5 $\mathrm{nm}$ [13], allowing orientation mapping on an extremely fine scale. The DTE test method and gas gun system used is described elsewhere [9, 14], and details of the EBSD, TKD and transmission electron microscopy (TEM) parameters, as well as specimen preparation are provided in $[9,15]$. 
Figure 1(a) shows the segment left in the die after DTE testing at $400 \mathrm{~m} \mathrm{~s}^{-1}$. Material points along the symmetry axis of the part of the specimen left in the die after testing are representative of different instances along a common deformation history [9], and therefore the investigation was focused on such points in order to follow the microstructure evolution with progressive degree deformation, see Fig. 1(a).

The development of a dual $\langle 111\rangle+\langle 001\rangle$ fiber texture with increasing strain is shown in Fig. 1(b), which also includes the development of the measured recrystallized fraction (RX). From the inverse pole figure (IPF) map in Fig. 1(c), corresponding to region 4, individual recrystallized grains with different orientations can be seen among the elongated grains with fiber texture. The RX fraction was measured to around $7 \%$ in this region, see Fig. 1(d) where the recrystallized (RX), deformed (DF) and substructured (SS) grains are identified. In region 5 the recrystallized fraction, around $60 \%$, dominates the microstructure, and it is clear that the recrystallization texture is very different from that developed during deformation, see Fig. 1(e) and (f)).

The EBSD maps in Fig. 1 indicate the operation of a discontinuous recrystallization process. In order to further study the early stages of DRX, specimens for TEM/TKD were extracted from region 4. Figure 2(a) shows an example of such a TKD investigation, with the orientation map superimposed on the band contrast. From the band contrast, the deformation substructures can be clearly identified, and a distinct difference between the $\langle 001\rangle$ (red) and $\langle 111\rangle$ grains (blue) can be seen. The $\langle 001\rangle$ grains contain elongated microbands, around 200-300 nm wide and several $\mu \mathrm{m}$ long, parallel to the tensile axis. In contrast, a more equiaxed cell substructure is observed in the $\langle 111\rangle$ oriented regions, with cell sizes up to around $1 \mu \mathrm{m}$. This was also verified by TEM, as shown in the example in Fig. 2(b). Similar substructure features can be seen in the TKD map in Fig. 3(a), with a clear difference between the deformation substructures in the $\langle 001\rangle$ and $\langle 111\rangle$ grains.

The simultaneous presence of both equiaxed cells and microbands has been noted previously in DTE tested $\mathrm{Cu}[8]$, but not attributed to the orientation of the grain in which they are observed. When subjected to moderate tensile deformation, copper tends to show an equiaxed cell structure in the $\langle 001\rangle$ grains, and a diffuse, highly tangled dislocation structure with less well-defined cells and boundaries in the $\langle 111\rangle$ grains $[16,17]$. The difference in substructure in the $\langle 001\rangle$ fibre seen 
here, compared to the literature, could be related to the degree of deformation of the material, where a more severe deformation could convert the equiaxed structure of the $\langle 001\rangle$ grains to elongated cells or microbands. This is supported by the observation of elongated cells in the cube oriented grains of pure copper after rolling to an equivalent von Mises strain of 0.78 [17], which could be an intermediate stage in the conversion from cell structure to microbands. Misorientation along the white arrows marked A and B in Fig. 2(a) are shown in Fig. 2(c). The elongated subgrains in the $\langle 001\rangle$ region have a low internal misorientation (low density of dislocations), and sharp subgrain boundaries with a misorientation of around $1-3^{\circ}$. In contrast, the equiaxed substructure in the $\langle 111\rangle$ region is much more diffuse. Similar results are seen when comparing the misorientations along arrows A and B in Fig. 3(a), see first panel in Fig. 3(b). These results are consistent with previous results from TEM studies [16, 18], EBSD pattern quality analysis [19] and neutron diffraction [16, 20], which have also shown a higher dislocation density in $\langle 111\rangle$ oriented grains in drawn copper and aluminum wires. Comparison of the pattern quality for the fibers in the present case show similar results, with significantly higher quality for the $\langle 001\rangle$ fiber, consistent with a lower dislocation density. Calculations based on neutron diffraction data typically show that the stored energy in the deformed $\langle 111\rangle$ fiber is around twice that of the $\langle 001\rangle$ component in copper $[16,20]$.

Figure 3(c) is a close-up of the region in Fig. 3(a) indicated by a box, and shows the deviation from the ideal $\langle 001\rangle$ and $\langle 111\rangle$ orientations (the scale is limited to a deviation of less than $20^{\circ}$ from ideal, in order to reveal the details). A number of distinct subgrains with larger deviation can be seen in both $\langle 001\rangle$ and $\langle 111\rangle$ regions, primarily along the grain boundary. The occurrence of more equiaxed subgrains in the grain boundary region of the $\langle 001\rangle$ grains, which otherwise consist of microbands, can be explained by the occurrence of multiple slip in order to maintain strain compatibility with the adjacent grain. The misorientations along arrows $\mathrm{C}$ and $\mathrm{D}$ in the $\langle 001\rangle$ subgrains are shown in Fig. 3(b). The grain at arrow D has already (partially) formed a high angle boundary with misorientation above $10^{\circ}$ to the parent grain. Similarly, distinct subgrains with large deviations can be seen in the $\langle 111\rangle$ grains, see arrows E and F in Fig. 3(b) and (c). The misorientation gradients inside the subgrains in the $\langle 111\rangle$ grain are considerably smaller than outside, indicating a low internal dislocation density, compared to the tangled cells in the surrounding. 
The observed distinctly misoriented subgrains in Fig. 3(b), both $\langle 001\rangle$ and $\langle 111\rangle$ oriented, show signs of bulging into the adjacent grain. This suggests that they could act as nuclei for recrystallization through the Bailey-Hirsch mechanism [21], consistent with our previous suggestion [9]. According to this model, subgrains adjacent to a HAGB can rotate to a favorable orientation and consume the adjacent deformed grain, driven by the difference in dislocation density $\Delta \rho$. The critical diameter for a subgrain bulging, $d_{c}$, can be calculated as $d_{c}=8 \sigma / \mu \rho b^{2}$ [21], where $\sigma$ is the grain boundary energy, $b$ is the magnitude of the Burgers vector and $\mu$ is the shear modulus. The dislocation density in severely deformed copper have been measured to $1.5 \times 10^{15}-3 \times 10^{16} \mathrm{~m}^{-2}$ at large strains $[22,23]$, and it could be assumed that similar levels would be achieved during DTE. Assuming $\Delta \rho=5 \times 10^{15}-1 \times 10^{16} \mathrm{~m}^{-2}$, the critical diameter is estimated to around $200-400 \mathrm{~nm}$ at homologous temperatures around 0.5 . This is very close to the size of the subgrains observed in Fig. 3(c). The subsequent growth of such nuclei during deformation and post-test cooling can be estimated by integrating of the grain boundary migration velocity over the temperature history, $T(t)$, from finite element simulation of the DTE process [9] according to

$$
d(t)=d_{c}+\frac{\beta \delta \Omega_{m} D_{0, G B} \Delta \rho}{R} \int_{t=0}^{t} \frac{\mu(T(t))}{T(t)} \exp \left\{-\frac{Q_{G B}}{R T(t)}\right\} d t
$$

where $\beta$ is the effective fraction of the Turnbull estimate of the grain boundary mobility [24], $\delta$ is the grain boundary width, $\Omega \mathrm{m}$ is the atomic volume and $D_{0, G B}$ and $Q_{G B}$ are the pre-exponential and activation energy, respectively, for grain boundary diffusivity, $\mu$ is the shear modulus and $R$ is the gas constant. By including both heat generation during deformation and post-test cooling in the die (assuming perfect contact between specimen and die, and accounting for convection, radiation and conduction), a conservative estimate of the final grain size in region 5 is in the order of 10-25 $\mu \mathrm{m}$, depending on the size of the nucleus, as shown in Fig. 4. Clearly, very limited growth occurs during on-going deformation. Rather, the grain growth occurs during the post-deformation cooling phase. Although it is not possible to resolve from the present analysis, the hypothesis is that the nucleation process, in terms of the formation of the equiaxed subgrains in the $\langle 001\rangle$ grains, is a result of the increasing compatibility strains at the grain boundaries during deformation. The nucleation (subgrain formation) can therefore be considered as a result of dynamic recrystallization, even if the subsequent grain growth occurs during static conditions. 
Experimental observations in region 5 show that the large recrystallized grains are in the order of $10-40 \mu \mathrm{m}$, see Fig. 5, which is in good agreement with the grain growth model given the uncertainties in the model parameters and boundary conditions. Here the temperature dependence of the shear modulus and grain boundary diffusivity were considered, whereas the effect of temperature on the atomic volume was neglected. No pressure induced effects on the parameters were considered. For more information on the finite element simulations and grain growth model, including model parameters, see [9].

Although the distinct subgrains presumably acting as DRX nucleation sites are seen in both $\langle 001\rangle$ and $\langle 111\rangle$ grains, the change in relative texture strength in Fig. 1(b) indicates that the $\langle 111\rangle$ fiber is preferentially replaced during subsequent grain growth. This is more clearly seen from the IPFs for the different subsets (Fig. 6), which show that the deformed grains in region 4 are dominated by the $\langle 111\rangle$ fiber, whereas the RX subset mainly consists of $\langle 001\rangle$ oriented grains. However, in region 5 both DF and RX subsets have a clear $\langle 001\rangle$ dominance, and diffuse weak components. By calculating the fiber volumes (see Fig. 6), it can be shown that also the $\langle 001\rangle$ grains in the DF subsets are replaced during recrystallization. This is similar to the course of events suggested to occur during static recrystallization of drawn copper wire [25, 26]. Contrary to observations in aluminum [18], the subgrains in the $\langle 111\rangle$ grain are of similar size as those in $\langle 001\rangle$, ruling out the size advantage of the recrystallized nuclei as a reason for $\langle 001\rangle$ predominance during DRX. Another explanation for the $\langle 001\rangle$ dominated recrystallization texture in copper is based on the strain-energy release maximization model [27], where it is energetically favorable to replace deformed grains, both with $\langle 001\rangle$ and $\langle 111\rangle$ orientation, with $\langle 001\rangle$ grains as a results of the anisotropic elasticity of copper crystals. Baudin et al. [19], on the other hand, explained the recrystallization texture by the higher driving force for migration of $\langle 001\rangle$ boundaries due to the higher dislocation density in the $\langle 111\rangle$ grains. However, the present study does not allow us to discriminate between these mechanisms. Additionally, the high fraction of $\Sigma 3$ boundaries in the recrystallized microstructure $(\approx 50 \%$ in region 5 as can be seen in Fig. 7$)$ indicates that growth twinning plays a large role in the formation of the final texture. This can also be seen in the large fraction of orientations outside the main fibers in region 5 (see Fig. 6), which to a large degree is made up of twinned $\langle 001\rangle$ grains. 
In summary, the nucleation phase of dynamic recrystallization during high-strain-rate tensile deformation of pure copper was investigated by high-resolution orientation mapping using TKD. Individual subgrains with high misorientation were observed along the grain boundaries between $\langle 001\rangle$ and $\langle 111\rangle$ grains. They presumably form due to compatibility strains at the boundaries between adjacent grains during deformation. The size of these subgrains was consistent with the estimated critical size for grain boundary bulging based on the Bailey-Hirsch mechanism. Considering the predicted grain growth of such nuclei during adiabatic heating and postdeformation cooling, the observed subgrains sizes are also consistent with the final recrystallized microstructure. Based on this, it is proposed that nucleation of dynamic recrystallization during high-strain-rate tensile deformation takes place by similar mechanisms as during quasi-static conditions, in contrast to the rotational recrystallization observed during dynamic shear or shearcompression testing with forced strain localization. Thus, it is possible that DRX models based on quasi-static testing could be extended to include dynamic deformation in the case of tensile loading. 


\section{References}

[1] F. J. Humphreys and M. Hatherly: Recrystallization and Related Annealing Phenomena, $2^{\text {nd }}$ ed., Elsevier Ltd., Kidlington, 2004.

[2] Y. Meshcheyakov, S. Atroshenko, A. Divakov and N Naumova: AIP Conf. Proc., 2012, vol 1426, pp. 1367-1370.

[3] U. Andrade, M.A. Meyers, K.S. Vecchio and A.H. Chokshi: Acta Metall. Mater., 1994, vol 42, pp. 3183-3195.

[4] J.A. Hines and K.S. Vecchio: Acta Mater., 1997, vol. 45, pp. 635-649.

[5] M. A. Meyers, Y. B. Xu, Q. Xue, M. T. Pérez-Prado and T. R. McNelley: Acta Mater., 2003, vol. 51, pp. 1307-1325.

[6] L. Tang, Z. Chen, C. Zhan, X. Yang, C. Liu and H. Cai: Mater. Charact., 2012, vol 64, pp. 21-26.

[7] D. Rittel, P. Landau and A. Venkert: Phys. Rev. Lett., 2008, vol 101, p. 165501.

[8] G.T. Gray III, E.K. Cerreta, C.A. Yablinsky, L.B. Addessio, B.L. Henrie, B.H. Sencer, M. Burkett, P.J. Maudlin, S.A. Maloy, C.P. Trujillo and M.F. Lopez: AIP Conf. Proc., 2006, vol 845, pp. $725-728$.

[9] M. Hörnqvist, N. Mortazavi, M. Halvarsson, A. Ruggiero, G. Iannitti and N. Bonora: Acta Mater., 2015, vol 89, pp. 163-180.

[10] A.H. Chokshi and M. Meyers: Scripta Metall. Mater., 1990, vol. 24, pp. 605-210.

[11] D. Rittel: J. Phys. D, 2009, vol 42, p. 214009.

[12] S. Osovski, D. Rittel and A. Venkert: Mech. Mater., 2013, vol. 56, pp. 11-22.

[13] P.W. Trimby: Ultramicr., 2012, vol.120, pp. 16-24.

[14] N. Bonora, G. Testa, A. Ruggiero, G. Iannitti, N. Mortazavi and M. Hörnqvist: J. Dyn. 
Behav. Mater., 2015, vol. 1, pp. 136-152.

[15] N. Mortazavi, M. Esmaily and M. Halvarsson: Mater. Lett., 2015, vol. 147, pp. 42-45.

[16] S. Jakani, T. Baudin, C.H. de Novion and M.H. Mathon: Mater. Sci. Eng. A, 2007, vol. 456, pp. 261-269.

[17] X. Huang and G. Winther: Phil. Mag., 2007, vol. 87, pp. 5189-5214.

[18] H. Inoue, N. Inakazu and H. Yamamoto: Proc. $6^{\text {th }}$ Int. Conf. Texture of Materials, pp. 591-600, The Iron and Steel Institute of Japan, 1981.

[19] T. Baudin, A.L. Etter and R. Penelle: Mater. Charact., 2007, vol. 58, pp. 947-952.

[20] S. Jakani, M.H. Mathon, P. Benyoucef, M.and Gerber, T. Baudin and C.H. de Novion: J. Neutron Res., 2004, vol. 12, pp. 249-254.

[21] J.E. Bailey and P.B. Hirsch: Proc. Royal Soc. London A, 1962, vol. 267, pp. 11-30.

[22] J. Gubicza, L. Balogh, R.J. Hellmig, Y. Estrin and T. Ungár: Mater. Sci. Eng. A, 2005, vol. 400-401, pp. 334-338.

[23] Yoshinori Murata, Ippei Nakaya and Masahiko Morinaga: Mater. Trans., 2008, vol. 49, pp, 20-23.

[24] D. Turnbull: Trans. AIME, 1951, vol. 191, pp. 661- 665.

[25] P. Gerber, S. Jakani, T. Baudin, M.H. Mathon and R. Penelle: Mater. Sci. Forum, 2004, vol. 467-470, pp. 135-140.

[26] H. Park and D.N. Lee: Metall. Mater. Trans. A, 2003, vol. 34A, pp. 531-541.

[27] D.N. Lee: Scripta Metall. Mater., 1995, vol. 32, pp. 1689-1694. 

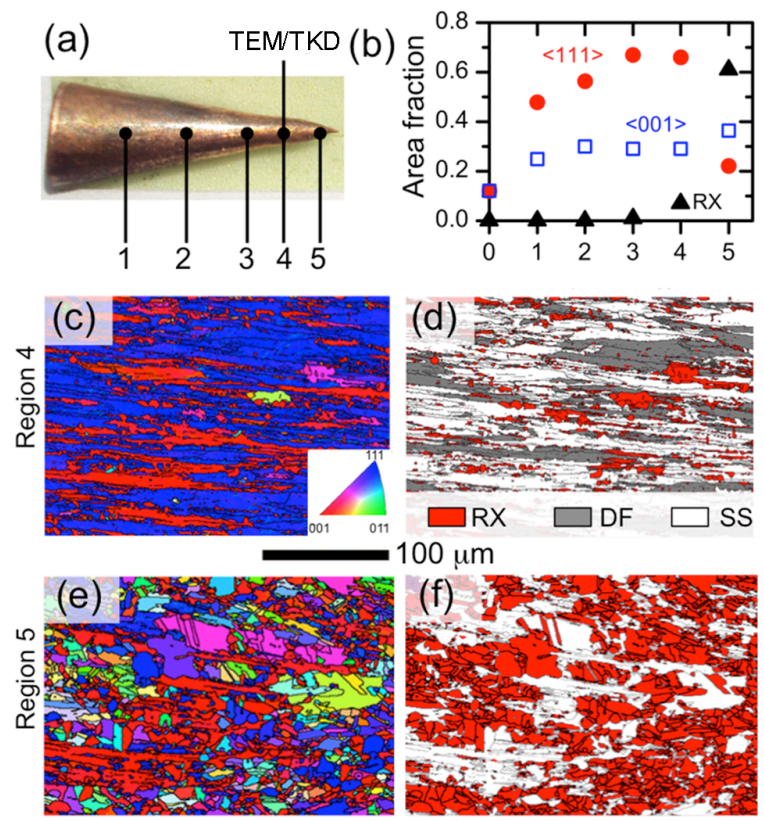

Figure 1: (a) Segment left in the die after DTE testing. The numbers indicate the positions along the symmetry axis where EBSD and TKD investigations were performed; (b) Measured area fractions of the $\langle 111\rangle$ and $\langle 001\rangle$ fibers, and recrystallized fraction (RX) for the different positions in (a). (c) IPF map of position 4, showing an elongated grain structure aligned with the extrusion direction. (d) Map of recrystallized (RX), deformed (DF) and substructured (SS) grains in region 4. (d) IPF map of position 5, and (e) corresponding RX/DF/SS map showing a partially recrystallized microstructure after grain growth during cooling.
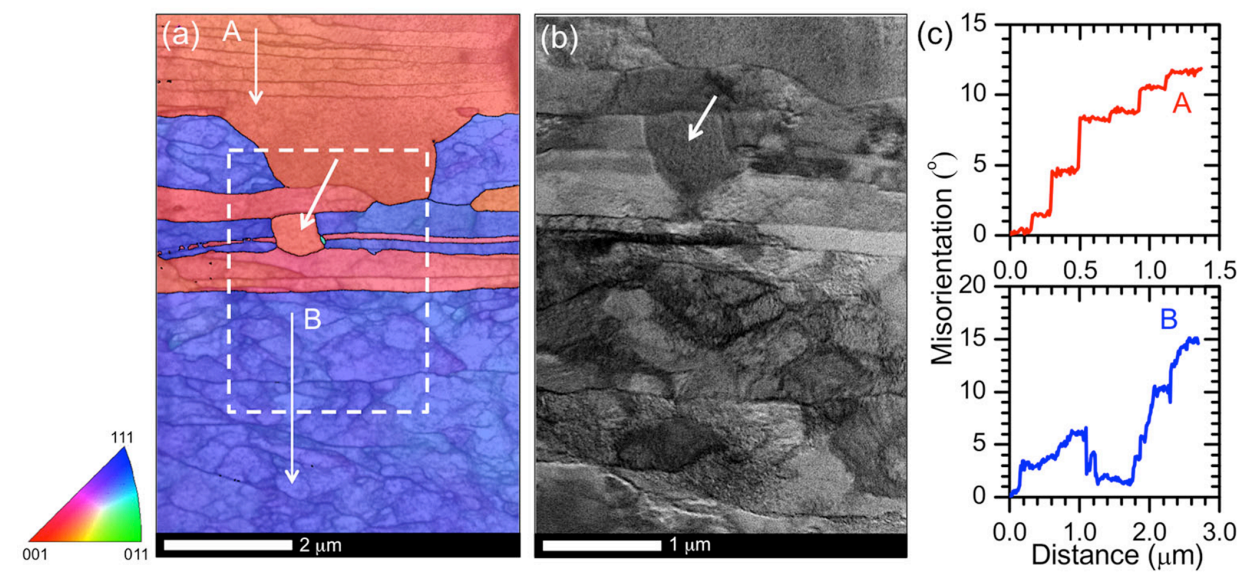

Figure 2: (a) TKD IPF map, superposed on a band contrast map. (b) BF TEM image of the boxed region in (a), showing the substructure difference between $\langle 001\rangle$ and $\langle 111\rangle$ grains. The slanted arrow points to the same recrystallized grain as in (a). (c) Misorientation profiles along arrows $\mathrm{A}$ and $\mathrm{B}$ in (a). 

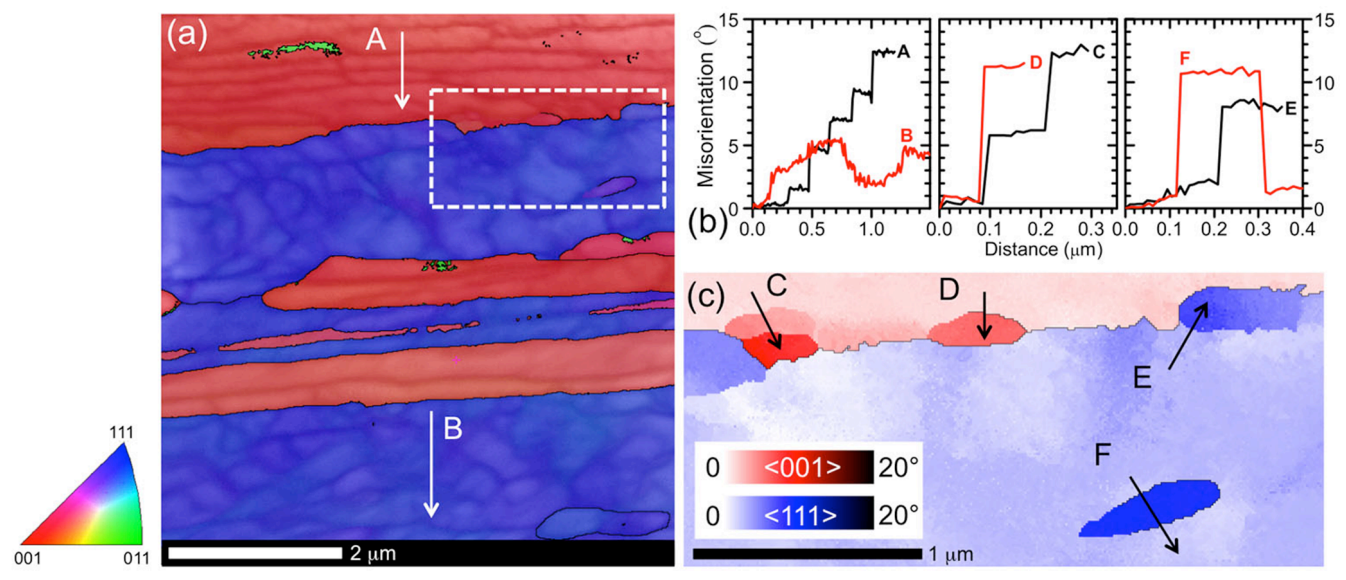

Figure 3: TKD IPF map, superposed on a band contrast map. (b) Misorientation profiles along the lines indicated in (a) and (c). (c) Magnification of the boxed region in (a), with the deviation from the ideal $\langle 001\rangle$ and $\langle 111\rangle$ orientations is shown.

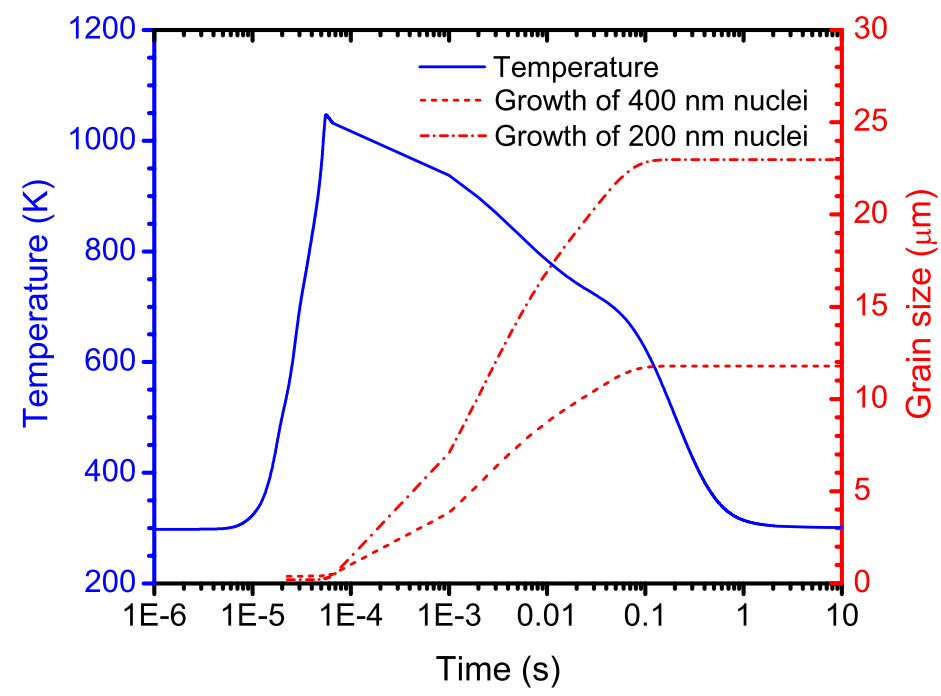

Figure 4: Temperature history in region 5 from finite element simulations and corresponding calculated growth of recrystallized nuclei during the DTE test and post-test cooling. 


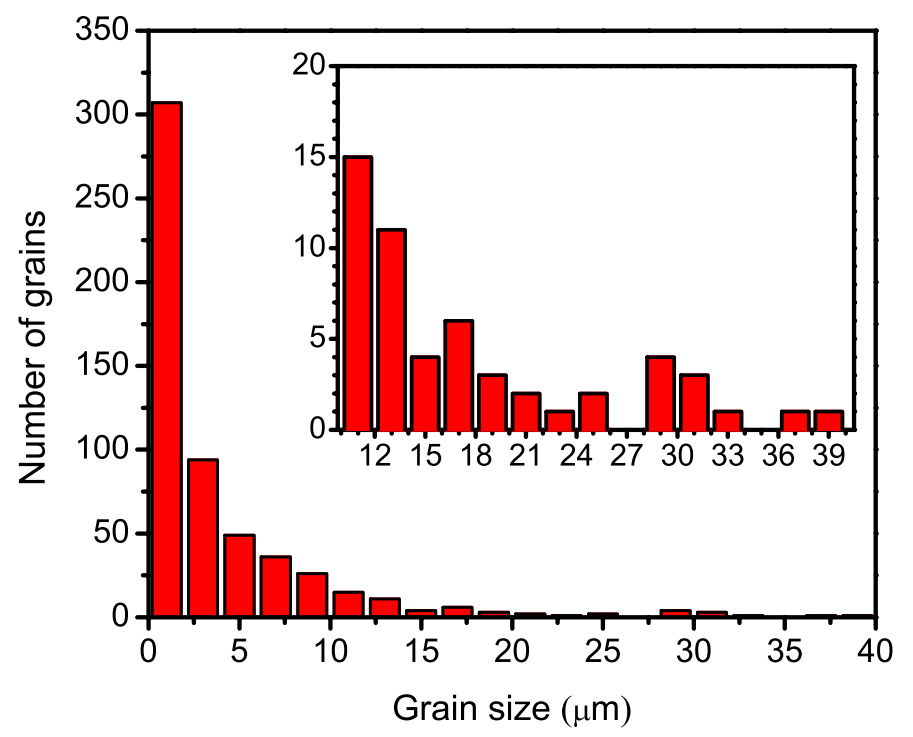

Figure 5: Final grain size distribution in the recrystallized (RX) fraction in region 5. Insert shows a magnification of the part of the distribution between 10 and $40 \mu \mathrm{m}$.

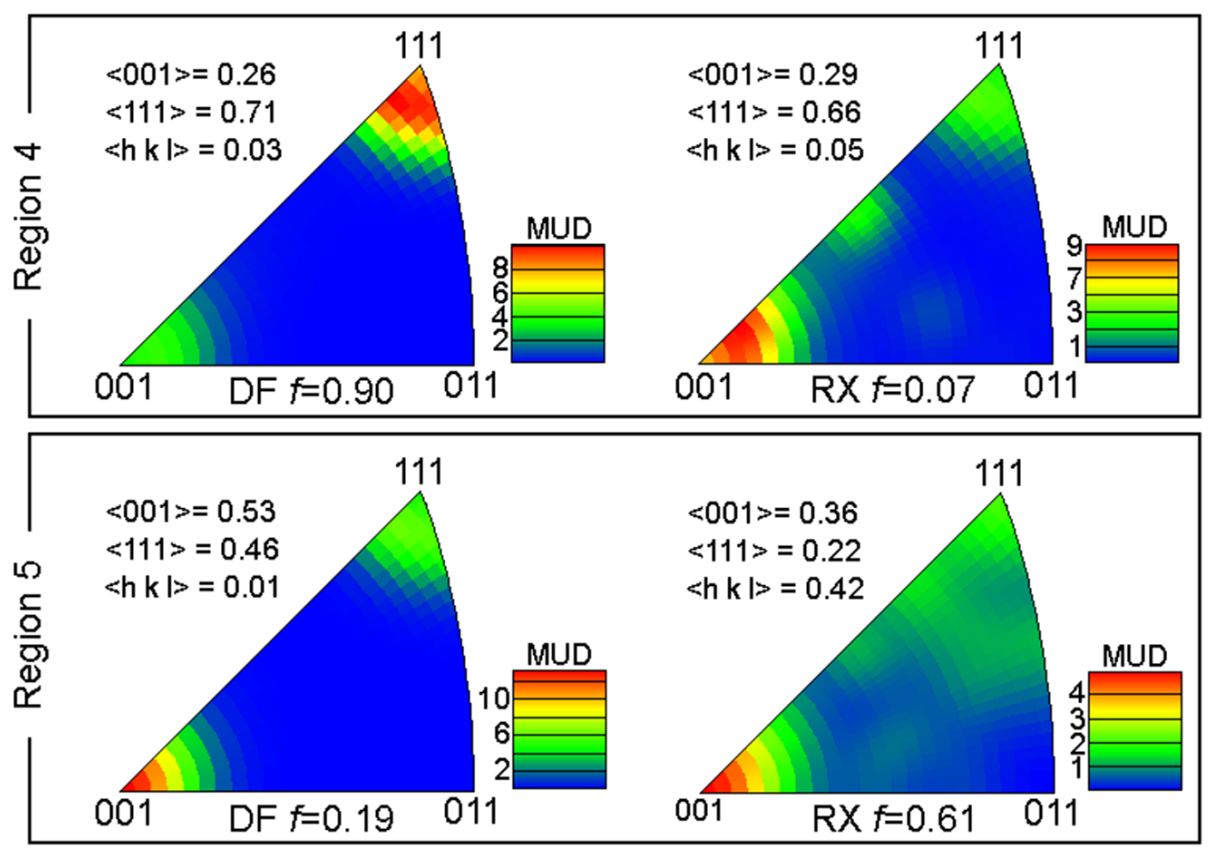

Figure 6: IPFs showing the textures in the deformed (DF) and recrystallized (RX) subsets in region 4 and 5 (MUD=multiples of uniform distribution). $f$ denotes the fraction of the subsets, and the numbers for $\langle 001\rangle,\langle 111\rangle$ and $\langle\mathrm{hkl}\rangle$ refers to the fraction of the respective fibers in each subset ( $\langle\mathrm{hkl}\rangle$ refers to orientations outside the two main fibers). 


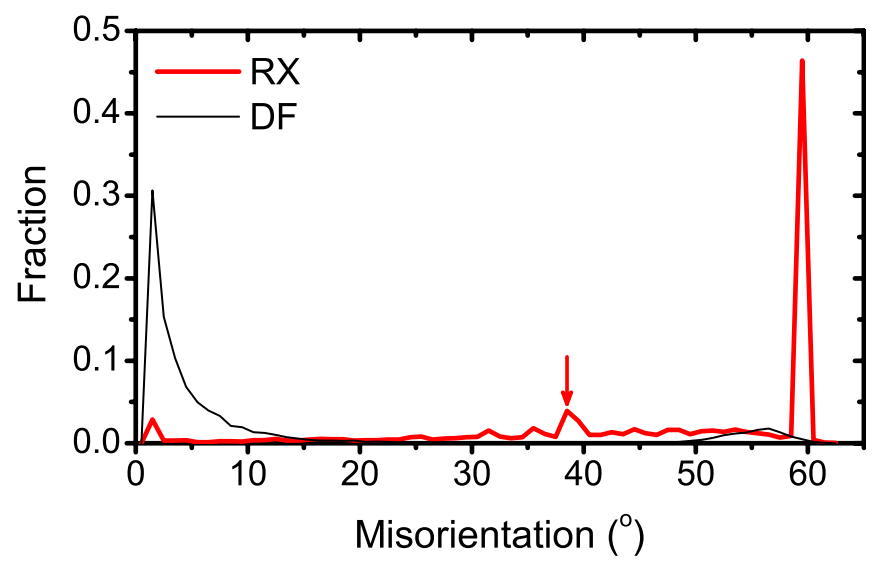

Figure 7: Grain boundary misorientation distribution in the recrystallized (RX) and deformed (DF) subsets in region 5 , showing a large fraction of twin boundaries $\left(\Sigma 3, \theta=60^{\circ}\right)$ in the RX subset. The arrow indicates the presence of secondary twins $(\Sigma 9)$. 